\title{
A Perspectiva do Paciente no Roteiro de Anamnese: o Olhar do Estudante
}

\author{
The Patient's Perspective in the Anamnese \\ Script: the Student's View
}

\author{
Paula Martins Balduino ${ }^{I}$ \\ Fernando Plazzi Palis \\ Vinícius Ferreira Paranaíba \\ Helcia Oliveira de Almeida ${ }^{I}$ \\ Eliana Mendonça Vilar Trindade
}

\author{
PALAVRAS-CHAVE \\ - Empatia. \\ - Relações Médico-Paciente. \\ - Anamnese \\ - Educação Médica
}

Recebido em: 20/04/2012

Aprovado em: 30/05/2012

\section{RESUMO}

Introdução: Para possibilitar que o estudante visualize a doença de forma plena e integrada à história de vida da pessoa, foram incluídas no roteiro de anamnese questões relativas à subjetividade do paciente. Objetivo: Investigar as percepções dos estudantes da primeira à terceira série do curso de Medicina da Escola Superior de Ciências da Saúde acerca de questões relativas às perspectivas do paciente. Metodologia: Estudo descritivo, de cunho quantitativo e qualitativo, exploratório e transversal. Os dados foram coletados por meio de um questionário estruturado aplicado a uma amostra constituída por 60 estudantes, complementado por uma entrevista semiestruturada, realizada com uma amostra constituída por dez estudantes. Resultados: Sessenta e nove por cento dos estudantes se sentem frequentemente à vontade para perguntar sobre as perspectivas do paciente e $47,3 \%$ raramente se sentem preparados para acolher o paciente diante dos sentimentos mobilizados pelo item. Conclusão: $A$ inclusão no roteiro de anamnese de questões relativas às perspectivas do paciente foi positiva, ainda que insuficiente para aprofundar os vínculos empáticos, o que indica a necessidade de maior atenção ao desenvolvimento e consolidação de habilidades afetivas e empáticas no estudante. Observou-se também a persistência das dificuldades inerentes à inclusão da subjetividade do paciente durante a anamnese, o que pode agravar o risco de erosão da empatia ao longo do curso.

\section{ABSTRACT}

Introduction: To enable the student to view the disease integrated into the person's life story, questions related to the patient's subjectivity were included in the anamnese script. Objective: Investigate the perceptions of students from 1st to 3rd grades of the Medical School of Escola Superior de Ciências da Saúde related to patient's perspectives. Methodology: Descriptive, quantitative, qualitative, exploratory and transversal study. Data were collected using a structured questionnaire applied to a sample of 60 students and complemented by a semi-structured interview conducted with a sample of ten students. Results: $69 \%$ of the students often feel free to ask about the patient's perspectives and $47.3 \%$ rarely feel prepared to accept the patient face of the feelings mobilized by the item. Conclusion: The inclusion of questions related to the patient's perspectives in the anamnese script was positive, even that not sufficient to create empathy bonds, which indicates the need for greater attention to the development and consolidation of emotional and empathic skills in students. We also observed the persistence of the difficulties inherent to the inclusion of the patient's subjectivities during the interview, which may increase the risk of erosion of empathy during the course. 


\section{INTRODUÇÃO}

Nas últimas décadas, modificações no perfil de morbi-mortalidade e a avalanche de novas informações têm levado a profundas reflexões sobre a temática da educação médica ${ }^{1}$. A formação centrada na interpretação de exames cada vez mais complexos e na prescrição de drogas potentes não responde aos anseios dos pacientes, que querem entender e dar significado às próprias histórias ${ }^{2}$. Considerar a perspectiva da pessoa abre novas possibilidades de interação e traz o benefício do exercício de uma prática médica mais humanizada, pautada na autonomia do paciente e de seus familiares ${ }^{3}$.

O curso de Medicina da Escola Superior de Ciências da Saúde (ESCS) tem em sua estrutura curricular o programa educacional Habilidades e Atitudes Profissionais (HA), voltado para o desenvolvimento de habilidades e competências em Semiologia e Comunicação Médica, dentro de uma visão biopsicossocial, sendo realizado de forma sistematizada nas três primeiras séries. Busca-se possibilitar ao estudante a aquisição de competências relevantes de forma estruturada e progressiva $^{4}$.

Para visualizar a doença de forma plena e integrada à história de vida do paciente ${ }^{5}$, o estudante deve ser capaz de realizar uma anamnese abrangente, coletando informações, formulando adequadamente as perguntas, refletindo sobre o impacto das questões e sobre a qualidade da relação com o paciente $^{3}$

O termo anamnese se origina de ana = trazer de volta, recordar e mnese $=$ memória. Significa trazer de volta à mente os fatos relacionados com a pessoa e suas manifestações de doença. A anamnese tem como um de seus fundamentos o alcance de uma boa relação médico-paciente, que objetiva o vínculo, a adesão ao tratamento, a confiança e maior fidedignidade das informações prestadas pelo paciente ${ }^{6}$.

As informações colhidas sobre questões referentes à subjetividade do paciente contribuem para uma interpretação melhor dos sintomas pelo médico, para melhorar seu relacionamento com o paciente e para reduzir o sofrimento deste ${ }^{7}$, podendo ainda ser de grande ajuda para desfazer associações inapropriadas ou redirecionar o raciocínio clínico do profissional ${ }^{8}$.

Reflexões sobre a prática educacional nas diferentes séries evidenciaram que o modelo de aprendizagem adotado no programa educacional Habilidades e Atitudes, ainda que inovador, apenas descrevia e discutia o processo da entrevista, enquanto o conteúdo se restringia à história clínica. Ou seja, era oferecido aos estudantes um modelo para a formação de vínculo com o paciente, para a compreensão das percepções e vivências do paciente, para a correta condução da entrevista, enquanto o roteiro utilizado não abrangia essas dimensões.

Assim, decidiu-se incluir no roteiro de anamnese da ESCS temas relativos a perspectivas do paciente: (a) repercussões da doença sobre a vida, família e trabalho; (b) experiências semelhantes vivenciadas pelo paciente ou por pessoas próximas; (c) conselhos recebidos para lidar com a doença; (d) medidas tomadas pelo paciente para lidar com a doença; (e) principais preocupações atuais; (f) a que atribui a doença (causas); (g) como se sente com relação à doença; (h) percepções sobre o tratamento: dificuldades e benefícios; (i) percepções sobre o médico, a equipe de saúde e o atendimento; (j) dúvidas ou assuntos que o paciente julgar relevantes.

Este estudo descritivo transversal tem como objetivo investigar as percepções dos estudantes da primeira à terceira série do curso de Medicina da ESCS acerca das questões relativas às perspectivas do paciente incluídas no roteiro de anamnese. A revisão bibliográfica foi realizada por meio dos bancos de dados Bireme e Pubmed, com os seguintes critérios de inclusão: estudos quantitativos e qualitativos que envolvam a formação médica contemporânea e a relação médico-paciente e estudos que abordem o desenvolvimento de capacidades que se expressam em uma melhor comunicação estudante-paciente.

\section{METODOLOGIA}

\section{Sujeitos}

O estudo, desenvolvido na Escola Superior de Ciências da Saúde (ESCS), é de cunho quantitativo e qualitativo, exploratório e transversal. A amostra utilizada se baseou no critério de conveniência, pois o estudo previa a triangulação de métodos com a inclusão de abordagem qualitativa. Houve o cuidado de selecionar uma amostra que fosse significativa e emblemática do universo estudado. Ressalte-se a preocupação de incluir na amostra qualitativa sujeitos considerados informantes-chave e que preenchessem o critério da diversidade de tipos e da saturação teórica. $\mathrm{O}$ uso da triangulação de métodos se justifica pela possibilidade de combinar múltiplas estratégias de pesquisa, capazes de apreender as dimensões qualitativas e quantitativas do objeto e de garantir a representatividade e a diversidade de posições dos sujeitos que formam o universo da pesquisa quanto às ambições do método quantitativo, ao propiciar o conhecimento da magnitude, cobertura e eficiência da realidade estudada9 .

Para a aplicação de questionário estruturado, a amostra foi constituída por um grupo de 20 estudantes de cada uma das três primeiras séries do curso, somando um total de 60 
discentes. Para a entrevista semiestruturada, a amostra foi constituída por cinco estudantes da segunda série e cinco da terceira.

O critério de inclusão foi a disponibilidade do discente e o fato de estar cursando as três primeiras séries do curso de graduação em Medicina da ESCS. Foram excluídos os estudantes que se negaram a participar e os que responderam inadequadamente os questionários.

\section{INSTRUMENTOS}

a) Questionário estruturado, elaborado especificamente para este estudo, composto por perguntas fechadas, organizadas sob a forma de uma escala de tipo Likert, com o objetivo de investigar a percepção dos estudantes sobre a inserção da subjetividade do paciente no roteiro de anamnese;

b) Entrevista semiestruturada, com perguntas abertas acerca dos desafios e dificuldades inerentes à inclusão da perspectiva do paciente durante a anamnese. As perguntas abertas foram as seguintes: Qual a sua opinião sobre o item que aborda a perspectiva do paciente? Você se sente preparado para lidar com os sentimentos despertados com o item sobre a perspectiva do paciente?

\section{PROCEDIMENTO}

Os pesquisadores aplicaram o questionário estruturado e realizaram a entrevista semiestruturada de forma individual com os discentes em ambiente acadêmico, fora de horários letivos, durante o segundo semestre de 2008. Para garantir o respaldo das Diretrizes e Normas Regulamentadoras de Pesquisas Envolvendo Seres Humanos do Conselho Nacional de Saúde, foi aplicado antes dos questionários o termo de consentimento livre e esclarecido. Além disso, os pesquisadores, ao convidarem os estudantes para participar da pesquisa, enfatizaram o anonimato e explicitaram a finalidade do trabalho.

$\mathrm{Na}$ análise dos resultados foi utilizado o programa SPSS Versão 15.0.

\section{RESULTADOS}

\section{Análise Quantitativa dos Dados}

Mais de $50 \%$ dos estudantes responderam de forma positiva aos benefícios do item que aborda questões relativas às perspectivas do paciente incluídas no roteiro de anamnese. A grande maioria relatou se sentir à vontade de forma frequente ou muito frequente em perguntar sobre a perspectiva do paciente, e mais de $50 \%$ acreditam nos benefícios do item para a qualificação da relação estudante-paciente e para o aperfeiçoamento da comunicação (Tabela 1).
TABELA 1

Q1 - Você se sente à vontade ao perguntar quanto às perspectivas do paciente sobre a doença?

\begin{tabular}{cccccc}
\hline Série & $\begin{array}{c}\text { Nunca } \\
(\%)\end{array}$ & $\begin{array}{c}\text { Rara- } \\
\text { mente } \\
(\mathbf{\%})\end{array}$ & $\begin{array}{c}\text { Às vezes } \\
\mathbf{( \% )}\end{array}$ & $\begin{array}{c}\text { Frequen- } \\
\text { temente } \\
\mathbf{( \% )}\end{array}$ & $\begin{array}{c}\text { Muito } \\
\text { frequen- } \\
\text { temente } \\
\mathbf{( \% )}\end{array}$ \\
\hline $1^{\underline{a}}$ & 0 & 12,5 & 6,25 & 31,25 & 50 \\
$2^{\underline{a}}$ & 0 & 10 & 25 & 40 & 25 \\
$3^{\text {a }}$ & 5,25 & 15,75 & 15,75 & 42,25 & 21 \\
\hline $\mathbf{Q}^{2}-$ As questões relativas às perspectivas do paciente sobre a \\
doença funcionam como recurso de aproximação com o paciente?
\end{tabular}

Quando questionados quanto à contribuição das questões relativas às perspectivas do paciente para o desenvolvimento da habilidade de empatia, 68,75\% dos estudantes da primeira série avaliaram que isto frequentemente ou muito frequentemente ocorre, enquanto $31,25 \%$ avaliaram que isto só ocorre às vezes. Já para os estudantes da segunda série, 50\% deles avaliaram que isto frequentemente ou muito frequentemente ocorre, enquanto $25 \%$ avaliaram que isto só ocorre às vezes, e o mesmo percentual considerou que isto raramente ocorre. Os estudantes da terceira série consideram que essas questões frequentemente favorecem sua habilidade de empatia (63\%) e $21,25 \%$ consideram que isto só ocorre às vezes (Tabela 2 ).

TABELA 2

Q4 - Ao escutar o paciente no que se refere às suas emoções e perspectivas diante do adoecimento você se sente constrangido?

\begin{tabular}{cccccc}
\hline Série & $\begin{array}{c}\text { Nunca } \\
(\%)\end{array}$ & $\begin{array}{c}\text { Rara- } \\
\text { mente } \\
(\%)\end{array}$ & $\begin{array}{c}\text { Às vezes } \\
(\%)\end{array}$ & $\begin{array}{c}\text { Frequen- } \\
\text { temente } \\
(\%)\end{array}$ & $\begin{array}{c}\text { Muito } \\
\text { frequen- } \\
\text { temente } \\
(\%)\end{array}$ \\
\hline $1^{\underline{a}}$ & 12,5 & 43,75 & 37,5 & 6,25 & 0 \\
$2^{\mathbf{a}}$ & 20 & 60 & 20 & 0 & 0 \\
$3^{\mathbf{a}}$ & 5,25 & 58 & 21 & 10,5 & 5,25 \\
\hline
\end{tabular}

Q5 - As questões relativas às perspectivas do paciente sobre a doença favorecem sua habilidade de empatia?

\begin{tabular}{cccccc}
\hline $1^{\underline{\underline{a}}}$ & 0 & 0 & 31,25 & 31,25 & 37,5 \\
$2^{\underline{a}}$ & 0 & 25 & 25 & 40 & 10 \\
$3^{\underline{a}}$ & 0 & 15,75 & 21,25 & 52,5 & 10,5 \\
\hline
\end{tabular}


Dos estudantes entrevistados da primeira série, 56,25\% avaliam que frequentemente ou muito frequentemente as questões relativas às perspectivas do paciente sobre a doença propiciam a ele a livre expressão de sentimentos, 31,25\% acreditam que isto ocorre às vezes e $12,5 \%$ que apenas raramente ocorre. Embora a maioria dos estudantes entrevistados da segunda série (40\%) acredite que as questões frequentemente ou muito frequentemente propiciam ao paciente a livre expressão de sentimentos, $35 \%$ avaliaram que isto ocorre algumas vezes e $25 \%$ que apenas raramente isto ocorre. A maioria dos estudantes da terceira série $(42,25 \%)$ considera que somente às vezes propiciam a ele essa livre expressão de sentimentos.

Sobre a preparação do estudante para apoiar ou acolher o paciente diante dos sentimentos mobilizados ao falar sobre suas perspectivas, metade dos estudantes da primeira série diz que se sente às vezes preparada, $31,25 \%$ se sentem frequentemente ou muito frequentemente preparados e 18,75\% nunca ou raramente se sentem preparados. A maioria dos estudantes entrevistados da segunda série $(65 \%)$ apenas raramente se sente preparada, $25 \%$ se sentem às vezes preparados e apenas $10 \%$ se sentem frequentemente preparados. A maioria dos estudantes entrevistados da terceira série apenas raramente se sente preparada $(52,75 \%), 26,25 \%$ às vezes se sentem preparados e apenas $21 \%$ se sentem frequentemente preparados (Tabela 3).

TABELA 3

\begin{tabular}{|c|c|c|c|c|c|}
\hline \multicolumn{6}{|c|}{$\begin{array}{l}\text { Q6 - As questões relativas às perspectivas do paciente sobre a } \\
\text { doença propiciam a ele a livre expressão de sentimentos? }\end{array}$} \\
\hline Série & $\begin{array}{c}\text { Nunca } \\
(\%)\end{array}$ & $\begin{array}{l}\text { Rara- } \\
\text { mente } \\
(\%)\end{array}$ & $\begin{array}{l}\text { Às vezes } \\
(\%)\end{array}$ & $\begin{array}{l}\text { Frequen- } \\
\text { temente } \\
(\%)\end{array}$ & $\begin{array}{l}\text { Muito } \\
\text { frequen- } \\
\text { temente } \\
(\%)\end{array}$ \\
\hline $1^{\mathrm{a}}$ & 0 & 12,5 & 31,25 & 37,5 & 18,75 \\
\hline $2^{\underline{a}}$ & 0 & 25 & 35 & 35 & 5 \\
\hline $3^{\mathrm{a}}$ & 0 & 26,25 & 42,25 & 15,75 & 15,75 \\
\hline \multicolumn{6}{|c|}{$\begin{array}{c}\text { Q7 - Você se sente preparado para apoiar ou acolher o paciente } \\
\text { diante dos sentimentos mobilizados ao falar sobre suas } \\
\text { perspectivas? }\end{array}$} \\
\hline $1^{\text {a }}$ & 6,25 & 12,5 & 50 & $18,75 \%$ & 12,5 \\
\hline $2^{\mathrm{a}}$ & 5 & 60 & 25 & $10 \%$ & 0 \\
\hline $3^{\mathrm{a}}$ & 5,25 & 47,5 & 26,25 & $21 \%$ & 0 \\
\hline
\end{tabular}

Fazendo uma análise das três séries juntas, 69\% dos estudantes se sentem frequentemente à vontade para perguntar sobre as perspectivas do paciente, $52,7 \%$ acham que as perguntas sobre a perspectiva do paciente frequentemente funcionam como um recurso de aproximação e 49,1\% avaliam que frequentemente propiciam condições para aperfeiçoar a comunicação com o paciente. Para 56,4\%, essas questões frequentemente favorecem sua habilidade de empatia. A maioria dos estudantes $(41,8 \%)$ acredita que as questões frequentemente propiciam ao paciente a livre expressão de sentimentos e $36,4 \%$ avaliam que isso ocorre apenas algumas vezes. Por fim, 47,3\% dos estudantes raramente se sentem preparados para acolher o paciente diante dos sentimentos mobilizados ao falar sobre suas perspectivas.

\section{Análise Qualitativa dos Dados}

Foi realizada análise de conteúdo das respostas obtidas na entrevista semiestruturada, com o objetivo de interpretar os sentidos latentes apreendidos no discurso dos estudantes. A obtenção dos indicadores de sentido nos permitiu construir algumas categorias de análise ou zonas de sentido, conforme os pressupostos da Epistemologia qualitativa ${ }^{10}$.

Nesta perspectiva, valorizam-se tanto a relevância quanto a frequência de aparecimento dos termos. Segundo os princípios da proposta preconizada pela Epistemologia Narrativa, a produção científica consiste em uma forma de aproximação e diálogo com o real, visando a um conhecimento construtivo-interpretativo ${ }^{10}$.

A análise qualitativa dos dados foi crucial para corroborar os achados quantitativos, visto que a informação qualitativa é considerada válida por ser capaz de produzir um modelo de inteligibilidade diferenciado e validado pelos critérios oriundos da intersubjetividade na prática hermenêutica ${ }^{10}$.

Após a leitura flutuante e exaustiva do texto produzido, foi possível identificar os resultados descritos a seguir.

A maior parte dos discentes entrevistados, segundo a análise das respostas às questões abertas, acredita que a inclusão de um item específico sobre a perspectiva do paciente é importante e possibilita maior aproximação. Entretanto, nem sempre esse tema é abordado durante a anamnese. Estudantes relatam que às vezes o próprio paciente não se sente à vontade para desabafar e revelar de forma franca as suas perspectivas, e eventualmente o estudante não pergunta, para evitar incomodar o paciente.

A relação fica melhor, tem mais liberdade. Vai de cada paciente, tem paciente que não gosta de falar da vida pessoal. Às vezes fica trinta minutos falando, às vezes fica um minuto. Da última vez que perguntei sobre sua perspectiva sobre a doença, a paciente crônica disse: "já me acostumei a ficar em hospital, só me preocupa meu filho". É a hora em que o paciente fica mais comovido (estudante 4 , terceira série).

Na maioria das vezes consigo tirar algo do paciente. Uma vez senti que um paciente [...] mentiu. Concordo com a posição 
do item, durante a história você vai conquistando a confiança do paciente e depois pergunta. Em paciente retraído fazer a pergunta não faz diferença, ele se mantém retraído... (estudante 5 , segunda série).

É importante. Nem sempre pergunto, varia de paciente para paciente. Depende do bom senso, quando o paciente está revoltado é melhor não perguntar. Evito incomodar, como é o último item, pode ser cansativo". (estudante 1, terceira série).

Alguns discentes não se sentem preparados para formar efetivamente vínculos empáticos com os pacientes. O novo roteiro denuncia o desafio e as dificuldades inerentes à inclusão da subjetividade do paciente e do estabelecimento da empatia neste contexto.

[...] uma vez conversei com uma paciente que estava fazendo quimioterapia, ela começou a chorar, tentei mudar o assunto, toquei na paciente. Acho que para estabelecer vínculo vem da gente e do paciente, não do roteiro. Acho que discussões da comunicação ajudam. (estudante 4 , segunda série).

Não me sinto preparado. Por mais que tente me colocar no lugar da pessoa, não é a mesma coisa. Com morte não sei lidar. Uma vez um paciente chorou, ofereci água e tentei mudar de assunto. (estudante 2, segunda série).

A maioria dos estudantes relatou não se sentir preparada para lidar com os sentimentos despertados com o item sobre a perspectiva do paciente e muitos se sentem constrangidos na abordagem de temas subjetivos ligados ao universo emocional dos pacientes.

Ontem mesmo, a paciente começou a comentar com o filho sobre o irmão assassinado. Nessas horas uso da função fática. Acho que do jeito que eu faço não consola muito não. A tensão é o grande problema, os professores deviam deixar a gente mais tranquilo. Fico nervoso, dá branco. (estudante 5, terceira série)

[...] Se for muito drástico, não sei se tenho muita habilidade para lidar com isso. Uma vez um paciente chorou muito durante a anamnese, aí o tutor de comunicação assumiu. Quando um paciente manifesta sentimento, eu uso a reafirmação e concordo com ele. (estudante 3 , terceira série)

Por não se sentirem preparados, alguns estudantes podem assumir uma postura defensiva, tendendo a desvalorizar a subjetividade do paciente. Tal postura acaba por representar um boicote tácito ou mesmo explícito ao modelo biopsicossocial. Este modelo ainda não está totalmente consolidado entre o corpo discente e docente da ESCS, que por vezes não valoriza uma abordagem integrada do paciente.

\begin{abstract}
Acho muito difícil abordar a perspectiva do paciente, eu quero terminar minha história e vou perder tempo com a perspectiva. Acho importante, mas me sinto em uma situação de tensão, com a perspectiva fica mais difícil. O item sobre a perspectiva do paciente é como se fosse um apêndice. Se não for feito, vou ser avaliado sem problemas. (estudante 5 , terceira série)

[...] Não sei o que fazer quando pacientes falam de crenças, normalmente não emito opinião. Como não sei lidar, passo para o próximo assunto. [...] Por mais que a gente discuta textos na comunicação, na hora a experiência é sempre novidade. Não consigo resgatar textos e aplicar, por isso não vejo muita utilidade nessas discussões específicas. (estudante 2 , terceira série)
\end{abstract}

\section{DISCUSSÃO}

O presente estudo apresentou algumas limitações: tamanho amostral relativamente pequeno e não aleatório e ausência de uma "situação controle" em relação ao item introduzido no roteiro de anamnese. Contudo, o uso da triangulação de métodos garantiu a representatividade e a diversidade de posições dos sujeitos que formam o universo da pesquisa9.

A inserção do item perspectiva do paciente sobre a doença no roteiro de anamnese da ESCS parece contribuir para a aquisição da habilidade de empatia pelos estudantes. Isto é evidenciado pelos dados obtidos: mais da metade dos alunos questionados da primeira à terceira série responderam que o novo roteiro de anamnese favorece a conquista dessa habilidade.

Apesar disso, vários estudantes não se sentem preparados para formar vínculos significativos com os pacientes, o que foi corroborado pelas respostas às questões abertas. Tal ideia ressalta a importância da maturidade e da habilidade pessoal do entrevistador, que necessita desenvolver as capacidades de escuta, de manejar emoções negativas e de superar o medo, o constrangimento e a tensão.

A tendência dos currículos médicos atuais está direcionada para a formação humanística do profissional. Assim, a taxonomia de objetivos educacionais compreende os domínios cognitivo, afetivo e psicomotor ${ }^{11}$. A aquisição da habilidade de empatia pelo estudante de Medicina se enquadra, nesse contexto, no domínio afetivo. 
A palavra empatia tem origem grega, empatheia, que significa tendência para sentir o que se sentiria caso se estivesse na situação e circunstâncias experimentadas e vivenciadas por outra pessoa $^{12}$. Empatia tem sido também definida como a capacidade de entender aquilo que uma pessoa está sentindo e transmitir-lhe compreensão, mantendo ao mesmo tempo certa objetividade para poder prestar a ajuda necessária ${ }^{13}$.

A empatia é um importante elemento da formação médica. Seu papel no estabelecimento de uma boa relação entre pacientes e médicos é discutido em pesquisas sobre educação e cuidado médico. A empatia e a habilidade comunicacional aumentam a satisfação do paciente e sua confiança, e intensificam a técnica de diagnóstico e tratamento do médico ${ }^{14}$.

Muitas causas têm sido enumeradas para explicar as dificuldades dos estudantes em formar vínculos empáticos. Dentre elas estão a falta de tempo, a coleta automática dos dados contidos na anamnese e a influência de emoções do estudante $^{12}$.

No presente estudo, a falta de tempo durante a entrevista clínica não se justifica totalmente, pois os estudantes da primeira à terceira série realizam no máximo uma entrevista por semana e dispõem de tempo adequado para isso. Porém, o roteiro de anamnese requer grande quantidade de informações e podemos presumir que os estudantes priorizem a busca de sinais e sintomas, deixando de focalizar a dimensão psicossocial do paciente.

A fixação excessiva do estudante ao roteiro compromete a dinâmica inerente ao processo de encontro com o paciente. A entrevista passa a ser rígida, assumindo às vezes o caráter de um interrogatório maçante, o que impede uma comunicação mais plena, fluente e empática.

A maneira como é ensinada a semiologia, com a 'coleta' em lugar da 'construção' de uma história e a forma como são apresentados os casos clínicos, tanto na fase pré-clínica como nas visitas a leitos, valoriza predominantemente os dados biomédicos ${ }^{15}$ (p. 40).

Por outro lado, a influência das emoções do estudante pode explicar a dificuldade em formar o vínculo empático. As influências de mecanismos inconscientes ou parcialmente conscientes dos alunos durante a entrevista são visualizadas quando estes encontram situações incômodas vivenciadas em seus meios familiares e identificadas nas atitudes de certos pacientes ${ }^{12}$.

Segundo uma perspectiva psicanalítica, o médico deveria saber manejar a contratransferência, isto é, deveria saber lidar com a carga emocional eliciada pelo encontro com o paciente, que muitas vezes assume um valor simbólico ou mesmo reedi- ta situações conflitivas inconscientes do passado ${ }^{16}$. A preparação psicológica do futuro médico é uma necessidade. Vale ressaltar a importância dos grupos Balint, nos quais os participantes são convidados a refletir sobre conteúdos inconscientes inerentes à prática ${ }^{16}$.

O declínio da aprovação do item ocorre de forma leve e gradativa ao compararmos os estudantes do início do curso com os do terceiro ano. Isto coincide com achados recentes da literatura, reveladores do risco de declínio da empatia ao longo do curso nas escolas médicas, em particular no primeiro ano de contato com a realidade clínica ${ }^{17}$.

A literatura indica que o nível de empatia é mais sensível à mudança do que as habilidades comunicacionais. Tem sido ainda observado que grupos de estudantes com alta empatia desde o início do curso de Medicina tendiam à queda, enquanto estudantes que inicialmente demonstravam baixa empatia tendiam ao crescimento. A maioria dos estudantes parece se beneficiar com estratégias pedagógicas inovadoras, o que justifica esforços principalmente no que concerne à expressão da empatia ${ }^{18,19}$.

A dificuldade em atingir uma integração plena entre comunicação e semiologia e a persistência da coleta automática dos dados clínicos da anamnese levam a um questionamento sobre a capacidade do roteiro de integrar efetivamente essas habilidades. $\mathrm{O}$ estudante parece compreender que existem dois processos distintos: a abordagem subjetiva do paciente, que engloba o acolhimento e a formação do vínculo; e a abordagem objetiva, que compreende os dados clínicos do estado de saúde do paciente.

Um dos fatores que contribuem para a dificuldade de integrar a comunicação aos outros objetivos clínicos é o fato de que os estudantes se veem frente a dois modelos de entrevista médica, aparentemente conflitantes: um modelo, da comunicação, que descreve o processo da entrevista, e outro, a "história médica tradicional", que descreve o conteúdo da entrevista. Muito frequentemente, estudantes ignoram suas habilidades em comunicação, aprendendo e usando o roteiro tradicional como um guia não somente para o conteúdo, mas também como um processo de entrevista médica. Esse apego ao roteiro de anamnese faz com que os estudantes tendam a fazer questões fechadas, estruturando a entrevista de forma rígida, focada na pesquisa de informações biomédicas ${ }^{20}$, ou mesmo a fazer perguntas repetitivas ou que interrompam a fala do paciente, mudando completamente a direção dada por ele para o esclarecimento das questões do interesse do entrevistador ${ }^{21}$.

Docentes com formação tradicional tendem a privilegiar o domínio cognitivo, ou seja, a racionalização pura do conhecimento teórico, que, segundo a taxonomia de Morse e colaboradores, seriam as competências profissionais específicas, que 
incluem teoria, técnica e integração entre as duas anteriores, deixando de valorizar as competências adicionais, que são pensamento crítico, reconhecimento do contexto social, preocupação com os valores da profissão, entendimento da ética da profissão, entre outras ${ }^{11}$.

Para viabilizar uma abordagem que realmente integre habilidades comunicativas e clínicas, é necessário efetivar espaços onde docentes e discentes possam refletir sobre seus valores e paradigmas. Acreditamos que um programa de desenvolvimento docente que enfatize a educação permanente em saúde possa ser um caminho para ajudar na ressignificação dos conceitos sobre o conteúdo e o processo da anamnese ${ }^{22}$.

\section{CONSIDERAÇÕES FINAIS}

Estudantes das três primeiras séries do curso de Medicina da ESCS avaliam positivamente o novo formato de roteiro. Isto pode ser atribuído às características do currículo da ESCS, que busca inspiração no modelo biopsicossocial e na importância da formação humanística do estudante por meio de metodologias ativas.

O estudo exploratório demonstra a importância do interesse do estudante na perspectiva do paciente e na construção de uma relação de parceria. Entretanto, o declínio leve da aprovação do item pode indicar um risco de desgaste e de perda de empatia com o passar do tempo. Vários instrumentos têm sido desenvolvidos para medir a empatia ${ }^{23}$, em sua maioria enquanto medidas de autorrelato, entre os quais alguns são aplicáveis ao contexto médico ${ }^{24}$. Sugerimos novos estudos que possam avaliar o risco de erosão da empatia ao longo do curso.

Em nosso estudo, de forma indireta, observamos as dificuldades dos estudantes em desenvolver habilidades empáticas, sobretudo em sua dimensão afetiva.

A dificuldade de integrar efetivamente comunicação e semiologia prejudicou a obtenção, por parte dos estudantes, da visão global da entrevista, o que se reflete na capacidade de empatia. Enquanto o estudante não perceber a unicidade do processo e do conteúdo, a formação do vínculo será constantemente prejudicada pela necessidade que ele experimenta de obter a história patológica de forma objetiva.

Ainda que seja insuficiente para aprofundar o vínculo empático entre estudante e paciente, o novo roteiro de anamnese da ESCS contribui para melhorar a relação estudante-paciente, colaborando de forma significativa para o desafio de construir esse vínculo humanizado e empático.

O desenvolvimento de competências em comunicação não é apenas um instrumento para estabelecer uma relação mais estreita entre o paciente e o estudante; é um elemento importante para a coleta de uma anamnese mais completa.

\section{REFERÊNCIAS}

1. Mascia AR, Silva FB, Lucchese AC, De Marco MA, Martins MCFN, Martins LAN. Atitudes frente a aspectos relevantes da prática médica: estudo transversal randomizado com alunos de segundo e sexto anos. Rev Bras Educ Méd. 2009;33(1):40-48.

2. Grossman E, Cardoso MHCA. As narrativas em medicina: contribuições à prática clínica e ao ensino médico. Rev Bras Educ Méd. 2006;30(1):6-14.

3. Almeida HO, Alves NM, Costa MP, Trindade EMV, Muza GM. Desenvolvendo competências em comunicação: uma experiência com a medicina narrativa. Rev Bras Educ Méd. 2005;29(3):208-215.

4. Fundação de Ensino e Pesquisa em Ciências da Saúde. Projeto Pedagógico. Brasília, DF: [s.h.]; 2001.p.29;41.

5. Trindade EMV, Almeida HO, Novaes MR, Versiane ER. Resgatando a dimensão subjetiva e biopsicossocial da prática médica com estudantes de medicina: relato de caso. Rev Brás Educ Méd. 2005;29(1):48-50.

6. Santos JB. Ouvir o paciente: A anamnese no diagnóstico clínico. Brasília Médica. 1999;36(3):90-95.

7. López M. Anamnese. In: López M, Medeiros JL. Semiologia Médica: As bases do diagnóstico clínico. 5.ed. Rio de Janeiro: Revinter; 2004. p.23-38.

8. Dantas F, Ficher P, Rampes H. A Critical overview of homeopathy. Annals of internal medicine. 2003; 139(8): W73.

9. Minayo MCS, Assis SG, Souza ER, Luiza Garnelo, orgs. Avaliação por triangulação de métodos: abordagem de programas sociais. Rio de Janeiro: Editora Fiocruz; 2005.

10. González-Rey F. Pesquisa Qualitativa em Psicologia: Os Processos de Construção da Informação. São Paulo: Tompson; 2005.

11. Tapajós R. Objetivos educacionais na pedagogia das humanidades médicas: taxonomias alternativas (campos de significado e competência). Rev Bras Educ Méd. 2008;32(4):500-506

12. Takaki MH, Sant'Ana DMG. Empatia como essência no cuidado prestado ao cliente pela equipe de enfermagem de uma unidade básica de saúde. Cogitare Enferm. 2004;9(1):79-83.

13. Carraro TE, Radünz V. A empatia no relacionamento terapêutico: um instrumento do cuidado. Cogitare Enferm. 1996;1(2):50-52.

14. Davis MH. Measuring individual differences in empathy: evidence for a multidimensional approach. Journal of Personality and Social Psycholog.1983; 44:113-126.

15. Ribeiro MMF. Avaliação da atitude do estudante de Medicina da Universidade Federal de Minas Gerais, a respeito 
da relação médico-paciente, no decorrer do curso médico. Belo Horizonte; 2006. Doutorado [Tese] — Universidade Federal de Minas Gerais.

16. Mello Renata, Herzog R. Subjetividade e defesa na obra de Michael Balint. Revista mal-estar subjetivo. 2008; 8(4).

17. Hojat M, Vergare M, Maxwell K, Brainard G, Herrine S, Isenberg G, Veloski J, Gonnella J. The devil is in the third year: a longitudinal study of erosion of empathy in medical school. Academic Medicine. 2009; 84(9): 1182-1191.

18. Dereboy C, Harlak H, Gürel S, Gemalmaz A, Eskin M. Teaching empathy in medical education. Turkish Journal of Psychiatry. 2005; 16(2).

19. Winefield HR, Chur-Hansen A. Evaluating the outcome of communication skill teaching for entry-level medical students: does knowledge of empathy increase? Med Educ. 2000; 34:90-4.

20. Kurtz S, Silverman J, Benson J, Draper J. Marrying Content and Process in Clinical Method Teaching: Enhancing the Calgary-Cambridge Guides. Rev Academic Medicine. 2003;78(8):802-809.

21. Sucupira ACSL, Ferrer APS. Uma Experiência de Ensino de Propedêutica Pediátrica em Ambulatório. Pediatria. 2000;22(2):105-114.

22. Rovere MR. Gestion Estratégica de la Educacion Permanente em Salud in Education Permanente de Personal de Salud. In: Haddad J, Roschke MA, Davini MC. Educación permanente de personal de salud. Washington: Organización Panamericana de la Salud;1994. p.63-106. [Serie de Desarrollo de Recursos Humanos, 100]
23. May B, Alligood M. Basic empathy in older adults: conceptualization, measurement, and application. Issues in Mental Health Nursing.2000;21:375-386.

24. Hojat M, Gonnella J, Nasca T, Mangione S, Vergare M, Magee M. Physician Empathy: Definition, Components, Measurement, and Relationship to Gender and Specialty. Am J Psychiatry.2002; 159:1563-1569.

\section{CONTRIBUIÇÃO DOS AUTORES}

Paula Martins Balduino foi a principal responsável pela edição do texto final, além de contribuir nas outras etapas ( análise bibliográfica, coleta de dados e processamento dos dados). Fernando Plazzi Palis E Vinícius Ferreira Paranaíba ontribuíram durante todo o processo de execução da pesquisa em especial na análise de dados. Helcia Oliveira de Almeida, Eliana Mendonça Vilar Trindade orientaram todo o processo.

\section{CONFLITO DE INTERESSES}

Declarou não haver.

\section{ENDEREÇO PARA CORRESPONDÊNCIA}

Paula Martins Balduino

Escola Superior de Ciências da Saúde

SMHN Quadra 03 - conjunto A - Bloco 1 - Edifício Fepecs Brasília

CEP 70.710-907 — DF

E-mail: paulabadu@hotmail.com 